\title{
Extrait d'une lettre de Monsieur Ch. Hermite à Monsieur Paul Gordan.
}

..... En attendant, c'est des fractions continues algébriques que je prends la liberté de vous entretenir, ou plutôt d'une extension de cette théorie, ayant cherché le système des polynômes entiers en $x, U, V, W$, tels que le dévéloppement de l'expression à trois termes

$$
U \sin x+V \cos x+W
$$

commence par la plus haute puissance possible de la variable. Ces polynômes forment une série doublement infinie, ainsi que pouvait le. faire présumer l'analogie avec la théorie arithmétique des minima successifs de la quantité:

$$
x+a y+b z,
$$

où $a$ et $b$ sont des constantes numériques, $x, y, z$ des nombres entiers. Ces minima s'obtiennent en effet par la réduction continuelle de la forme quadratique ternaire:

$$
(x+a y+b z)^{2}+\frac{y^{2}}{\boldsymbol{a}}+\frac{z^{2}}{\beta},
$$

où entrent deux indéterminées $\alpha$ et $\beta$ auxquelles doivent être attribuées toutes les valeurs de zéro à l'infini. La première série conduira à la fraction continue de Lambert:

et s'obtient ainsi:

$$
\operatorname{tang} x=\frac{x}{1-\frac{x^{2}}{3-\frac{x^{2}}{5-}}}
$$

Soit $A=\sin x$, puis successivement:

$$
\begin{aligned}
& A_{1}=\int_{0}^{x} A x d x=\sin x-x \cos x, \\
& A_{2}=\int_{V}^{x} A_{1} x d x=\left(3-x^{2}\right) \sin x-3 x \cos x, \\
& A_{3}=\int_{V}^{x} A_{2} x d x=\left(15-6 x^{2}\right) \sin x-\left(15 x-x^{3}\right) \cos x,
\end{aligned}
$$


et en général: $A_{n+1}=\int_{0}^{x} A_{n} x d x$. Les formules élémentaires:

$$
\begin{aligned}
& \int \cos x F(x) d x=\sin x \mathfrak{F}(x)+\cos x F^{\prime}(x), \\
& \int \sin x F(x) d x=\sin x F^{\prime}(x)-\cos x \mathfrak{F}(x),
\end{aligned}
$$

où l'on suppose: $\boldsymbol{F}(\boldsymbol{x})$ un polynôme entier et:

$$
\mathfrak{F}(\boldsymbol{x})=\boldsymbol{F}(\boldsymbol{x})-\boldsymbol{F}^{\prime \prime}(\boldsymbol{x})+\boldsymbol{F}^{\mathrm{IV}}(\boldsymbol{x})-\cdots
$$

montrent que $A_{n}$ est de la forme $U \sin x+V \cos x, U$ et $V$ étant des polynômes entiers dont l'un est du degré $n$ et l'autre du degré $n-1$. En second lieu si l'on part du développement en série:

$$
A=\sin x=x-\frac{x^{3}}{2.3}+\frac{x^{5}}{2.3 .4 .5}-\cdots,
$$

on en conclura' aisément:

$$
A_{n}=\frac{x^{2 n+1}}{1.3 .5 \ldots 2 n+1}-\frac{x^{2 n+3}}{1.2 \times 3.5 \ldots 2 n+3}+\cdots
$$

ou encore :

$$
A_{n}=x^{2 n+1} \sum_{1} \frac{1}{(2 k+1)(2 k+3) \ldots(2 k+2 n+1)} \cdot \frac{(-1)^{k} x^{2 k}}{1.2 .3 \ldots 2 k} .
$$

Le premier terme de cette série étant en $x^{2 n+1}$, vous voyez que $U$ et $V$ sont bien les polynômes qui résultent de la théorie des fractions continues. Mais on peut $y$ parvenir par une autre voie.

Soit: $\mathfrak{A}=\frac{\sin x}{x}$, puis successivement:

$$
\begin{aligned}
& \mathfrak{A}_{1}=-\frac{1}{x} \frac{d \mathfrak{A}}{d x}=\frac{\sin x-x \cos x}{x^{3}}, \\
& \mathfrak{A}_{2}=-\frac{1}{x} \frac{d \mathfrak{A}_{1}}{d x}=\frac{\left(3-x^{2}\right) \sin x-3 x \cos x}{x^{5}}, \\
& \mathfrak{A}_{3}=-\frac{1}{x} \frac{d \mathfrak{A}_{2}}{d x}=\frac{\left(15-6 x^{2}\right) \sin x-\left(15 x-x^{3}\right) \cos x}{x^{7}}
\end{aligned}
$$

et en général: $\mathfrak{A}_{n+1}=-\frac{1}{x} \frac{d \mathfrak{A}_{n}}{d x}$. On reconnaît immédiatement qu'on aura

$$
\mathfrak{A}_{n}=\frac{U \sin x+V \cos x}{x^{2 n+1}}
$$

$U$ et $V$ étant encore des polynômes dont l'un est de degré $n$ et l'autre de degré $n-1$; on obtient aussi facilement la série:

$$
\mathfrak{A}_{n}=\frac{1}{1.3 .5 \ldots 2 n+1}-\frac{x^{2}}{2.3 .5 \ldots 2 n+3}+\cdots
$$


Il s'ensuil que: $\mathfrak{A}_{n}=\frac{A_{n}}{x^{2 n+1}}$; et par conséquent

$$
\frac{A_{n+1}}{x^{2 n+3}}=-\frac{1}{x} \frac{d}{d x}\left(\frac{A_{n}}{x^{2 n+1}}\right)
$$

c'est-à-dire :

$$
A_{n+1}=(2 n+1) A_{n}-\frac{d A_{n}}{d x} x
$$

mais $\frac{d A_{n}}{d x}=A_{n-1} x$, et nous parvenons entre trois termes consécutifs à la relation :

$$
A_{n+1}=(2 n+1) A_{n}-A_{n-1} x^{2} .
$$

De là se tire la fraction continue de Lambert, et l'équation différentielle des transcendantes de Bessel. Il suffit en effet d'observer que:

$$
A_{n-1}=\frac{1}{x} \frac{d A_{n}}{d x}, \quad A_{n-2}=\frac{1}{x^{2}}\left(\frac{d^{2} A_{n}}{d x^{2}}-\frac{1}{x} \frac{d A_{n}}{d x}\right)
$$

pour passer de l'égalité $A_{n}=(2 n-1) A_{n-1}-A_{n-2} x^{2}$ à celte équation si connue :

$$
\frac{d^{2} A_{n}}{d x^{2}}-\frac{2 n}{x} \frac{d A_{n}}{d x}+A_{n}=0,
$$

dont une seconde solution est donnée comme il est aisé de voir par la formule:

$$
A_{n}=U \cos x-V \sin x .
$$

Je vais maintenant sortir du domaine des fractions continues, et définir une seconde série de polynômes $U, V, W$ en posant:

$$
B_{n}=\int_{0}^{x} A_{n} d x
$$

puis successivement une troisième, une quatrième etc. par les relations semblables:

$$
C_{n}=\int_{0}^{x} B_{n} d x, \quad D_{n}=\int_{0}^{x} C_{n} d x, \text { etc. }
$$

Les formules déjà employées:

$$
\begin{aligned}
& \int \cos x F(x) d x=\sin x \mathfrak{F}(x)+\cos x F^{\prime}(x), \\
& \int \sin x F(x) d x=\sin x F^{\prime}(x)-\cos x \mathfrak{F}(x)
\end{aligned}
$$

donnent la composition de ces quantités, el montrent qu'en désignant par $\boldsymbol{P}_{n}$ le terme général de la série de rang $p$, on aura:

$$
P_{n}=U \sin x+V \cos x+W,
$$

Journal für Mathematik Bd. L.XXVI. Heft 4. 
$U$ et $V$ étant des polynômes entiers l'un du degré $n$, l'autre du degré $n-1$, et $W$ de degré $p-1$. Or le développement:

$$
P_{n}=x^{2 n+p} \sum_{0} \frac{(2 k+2)(2 k+4) \ldots(2 k+2 n)(-1)^{k} x^{2 k}}{1 \cdot 2.3 \ldots 2 k+2 n+p},
$$

dont le premier terme est de degré $2 n+p$, a bien la forme voulue. Ces mêmes quantités peuvent s'obtenir d'une autre manière comme il suit. Posons suivant que $p$ est pair ou impair:

$$
\mathfrak{P}=\frac{(-1)^{\frac{3}{2} p}}{x^{p}}\left[\cos x-1+\frac{x^{2}}{1.2}-\frac{x^{4}}{1.2 .3 .4}+\cdots+(-1)^{\frac{1}{2} p} \frac{x^{p-2}}{1.2 \ldots p-2}\right]
$$

ou bien:

$$
\mathfrak{P}=\frac{(-1)^{\frac{p-1}{2}}}{x^{p}}\left[\sin x-x+\frac{x^{3}}{1.2 .3}-\cdots+(-1)^{\frac{p-1}{2}} \frac{x^{p-2}}{1.2 \ldots p-2}\right]
$$

et faisons successivement:

$$
\mathfrak{P}_{1}=-\frac{1}{x} \frac{d \mathfrak{P}}{d x}, \quad \mathfrak{P}_{2}=-\frac{1}{x} \frac{d \mathfrak{P}_{1}}{d x}, \ldots \mathfrak{P}_{n+1}=-\frac{1}{x} \frac{d \mathfrak{P}_{n}}{d x} .
$$

Cette loi de formation donne très-facilement le développement en série de $\mathfrak{F}_{n}$, en partant du développement de $\mathfrak{Y}$, à savoir:

On retrouve ainsi :

$$
\mathfrak{P}=\frac{1}{1.2 \ldots p}-\frac{x^{2}}{1.2 \ldots p+2}+\frac{x^{4}}{1.2 \ldots p+4} \cdots
$$

$$
\mathfrak{P}_{n}=\sum_{0} \frac{(2 k+2)(2 k+4) \ldots(2 k+2 n)(-1)^{k} x^{2 k}}{1.2 .3 \ldots 2 k+2 n+p},
$$

ce qui conduit à la relation:

$$
\mathfrak{P}_{n}=\frac{\boldsymbol{P}_{n}}{\boldsymbol{x}^{2 n+p}}
$$

d'où l'on tire comme pour les quantités $A_{n}$, celle-ci:

$$
P_{n+1}=(2 n+p) P_{n}-\frac{d P_{n}}{d x} x .
$$

Mais la dérivée $\frac{d P_{n}}{d x}$ est le $n_{-}^{\mathrm{e}}$ terme de la $(p-1)^{\mathrm{e}}$ série, faisant donc:

$$
p=2,3,4, \ldots
$$

nous aurons successivement:

$$
\begin{aligned}
& B_{n+1}=(2 n+2) B_{n}-A_{n} x, \\
& C_{n+1}=(2 n+3) C_{n}-B_{n} x, \\
& D_{n+1}=(2 n+4) D_{n}-C_{n} x,
\end{aligned}
$$


J'ai calculé par ces formules et celles qui concernent $A_{n}$ les valeurs suivantes :

$$
\begin{aligned}
& A_{1}=\sin x-x \cos x, \\
& A_{2}=\left(3-x^{2}\right) \sin x-3 x \cos x, \\
& A_{3}=\left(15-6 x^{2}\right) \sin x-\left(15 x-x^{3}\right) \cos x \text {, } \\
& A_{4}=\left(105-45 x^{2}+x^{4}\right) \sin x-\left(105 x-10 x^{3}\right) \cos x \text {, } \\
& A_{5}=\left(945-420 x^{2}+15 x^{4}\right) \sin x-\left(945 x-105 x^{3}+x^{5}\right) \cos x, \\
& B_{0}=-\cos x+1 \\
& B_{1}=-x \sin x-2 \cos x+2 \text {, } \\
& B_{2}=-5 x \sin x-\left(8-x^{2}\right) \cos x+8 \text {, } \\
& B_{3}=-\left(33 x-x^{3}\right) \sin x-\left(48-9 x^{2}\right) \cos x+48, \\
& B_{4}=-\left(279 x-14 x^{3}\right) \sin x-\left(384-87 x^{2}+x^{4}\right) \cos x+384 \text {, } \\
& B_{5}=-\left(2895 x-185 x^{3}+x^{5}\right) \sin x-\left(3840-975 x^{2}+20 x^{4}\right) \cos x+3840, \\
& C_{0}=-\sin x+x \text {, } \\
& C_{1}=-3 \sin x+x \cos x+2 x, \\
& C_{2}=-\left(15-x^{2}\right) \sin x+7 x \cos x+8 x, \\
& C_{3}=-\left(105-12 x^{2}\right) \sin x+\left(57 x-x^{3}\right) \cos x+48 x \text {, } \\
& C_{4}=-\left(945-141 x^{2}+x^{4}\right) \sin x+\left(561 x-18 x^{3}\right) \cos x+384 x, \\
& D_{0}=\cos x-1+\frac{x^{2}}{2}, \\
& D_{1}=x \sin x+4 \cos x+x^{2}-4 \text {, } \\
& D_{2}=9 x \sin x+\left(24-x^{2}\right) \cos x+4 x^{2}-24 \text {. } \\
& D_{3}=\left(87 x-x^{3}\right) \sin x+\left(192-15 x^{2}\right) \cos x+24 x^{2}-192,
\end{aligned}
$$

C'est maintenant, Monsieur, que se présente une question arithmétique d'un grand intérêt. Supposons $x=i$, en faisant pour abréger:

$$
h=\frac{\sin i}{i}=\frac{e-e^{-1}}{2}, \quad h^{\prime}=\cos i=\frac{e+e^{-1}}{2} ;
$$

la quantité $P_{n}=U \sin x+V \cos x+W$ prendra la forme suivante

$$
i^{2 n+p}\left(u h+v h^{\prime}+w\right)
$$

où $u$ et $v$ sont toujours des nombres entiers, $w$ pouvant être fractionnaire, 
mais devenant également enlier quand $n$ croît au delà d'une certaine limite. On a en effet:

$$
W=-(-1)^{\frac{1}{2} p} \Sigma \frac{(p-k)(p-k+2) \ldots(p-k+2 n-2)(-1)^{\frac{4 k}{k}} x^{k}}{1.2 .3 \ldots k}
$$

en supposant $k=0,2,4, \ldots p-2$, si $p$ est pair et:

$$
W=(-1)^{\frac{p-1}{2}} \Sigma \frac{(p-k)(p-k+2) \ldots(p-k+2 n-2)(-1)^{\frac{k-1}{2}} x^{k}}{1.2 .3 \ldots k}
$$

en faisant $k=1,3,5, \ldots p-2$, si $p$ est impair; or dans les deux cas il est visible que le coefficient $\frac{(p-k)(p-k+2) \ldots(p-k+2 n-2)}{1.2 .3 \ldots k}$ finit par devenir entier. Cela posé, les divers systèmes des nombres

$$
x=u, \quad y=v, \quad z=w
$$

donneront-ils des minima de la fonction linéaire $x h+y k^{\prime}+z$ ?

Vous connaissez la découverte mémorable de Dirichlet sur les minima des fonctions linéaires, à un nombre quelconque d'indéterminées; en arithmétique elle me semble, si je puis dire aussi importante que la théorie des fonctions elliptiques pour l'analyse. Mais tandis que les fractions continues sont d'un emploi usuel, les applications numériques des théorèmes de Dirichlet, restent comme impossibles, et à cet ègard je reconnais n'avoir encore guère avancé la question, en déduisant ces théorèmes de la considération des formes quadratiques. Me plaçant toutefois en ce moment à mon point de vue, j'envisage les minima de la forme:

$$
f=\left(x h+y h^{\prime}+z\right)^{2}+\frac{x^{2}}{\alpha}+\frac{y^{2}}{\beta},
$$

où $\alpha$ et $\beta$ sont positifs et dont l'invariant est $D=\frac{1}{\alpha \beta}$. Ces minima satisfont à la condition $f \overline{\overline{2}} \sqrt[3]{2 D ;}$ or le produit $\left(h x+h^{\prime} y+z\right)^{2} \cdot \frac{x^{2}}{\alpha} \cdot \frac{y^{2}}{\beta}$ a pour maximum $\left(\frac{f}{3}\right)^{3}$, d'où cette relation indépendante de $\alpha$ et $\beta$, savoir:

$$
\left(h x+h^{\prime} y+z\right) x y<\sqrt{\frac{2}{2 y}} .
$$

En appliquant ce criterium aux nombres donnés par les quantités $B_{n}$, on reconnait immédiatement qu'ils ne peuvent convenir, mais dans les séries suivantes je trouve: 


$$
\begin{aligned}
i C_{2} & =16 h-7 h^{\prime}-8=\frac{1}{3.5 .6 .7}+\cdots \\
D_{2} & =-9 h+25 h^{\prime}-28=\frac{1}{3.5 .6 .7 .8}+\cdots \\
D_{3} & =-88 h+207 h^{\prime}-216=-\frac{1}{3.5 .7 .8 .9 .10}-\cdots \\
i E_{3} & =333 h-124 h^{\prime}-200=\frac{1}{3.5 .7 .8 .9 .10 .11}+\cdots \\
F_{3} & =166 h-501 h^{\prime}-678=\frac{1}{3.5 .7 .8 .9 .10 .11 .12}+\cdots \\
F_{4} & =2325 h-6136 h^{\prime}-6736=-\frac{1}{3.5 .7 .9 .10 \cdot 11.12 .13 .14}-\cdots
\end{aligned}
$$

etc., et vous voyez que la condition requise est complétement remplie, le calcul par logarithmes donnant dans le dernier cas:

$$
\frac{2325 \times 6136}{3.5 .7 .9 .10 .11 .12 .13 .14}=0,06283 \text {. }
$$

Mais je reviens à l'Algèbre, pour considérer les expressions rationnelles approchées de $\sin x$ et $\cos x$ données par deux équations telles que: $A_{n}=0$, $B_{n}=0$, ou bien $B_{n}=0, C_{n}=0 ; C_{n}=0, D_{n}=0$ etc. Dans le premier cas par exemple, on trouve pour $n=1,2,3$, ces valeurs:

$$
\begin{aligned}
& \sin x=\frac{2 x}{2+x^{2}},=\frac{24 x}{24+4 x^{2}+x^{4}},=\frac{720 x-48 x^{3}}{720+72 x^{2}+6 x^{4}+x^{6}}, \\
& \cos x=\frac{2}{2+x^{2}},=\frac{24-8 x^{2}}{24+4 x^{2}+x^{4}},=\frac{720-288 x^{2}}{720+72 x^{2}+6 x^{4}+x^{6}}
\end{aligned}
$$

et en général il est, aisé de voir qu'elles seront de la forme:

$$
\cos x=\frac{S}{R}, \quad \sin x=\frac{T}{R}
$$

$R, S$ et $T$ étant des polynòmes entiers dont les premiers renferment seulement des puissances paires et le troisième des puissances impaires de la variable. En déduisant d'abord des relations proposées:

$$
\cos x+i \sin x=\frac{S+i T}{R}
$$

j'observe que si l'on change $x$ en $-i x$, on se trouve amené à une expression entièrement réelle de l'exponentielle $e^{x}$, par une fraction dont le dénominateur ne contient que des puissances paires. Sous ce point de vue plus simple, je remarque qu'en posant:

$$
\Phi(x)=a_{10}+a_{1} x^{2}+a_{2} x^{4}+\cdots \cdot+a_{n} x^{2 n}
$$


on peut en général disposer des coefficients $a_{0}, a_{1}, \ldots$ de manière que le produit $e^{x} \boldsymbol{\Phi}(x)$ ordonné suivant les puissances croissantes de $x$, manque des $n$ termes en $x^{n+p+1}, x^{n+p+2}, \ldots x^{2 n+p}$, et soit de la forme:

$$
\boldsymbol{e}^{x} \boldsymbol{\Phi}(\boldsymbol{x})=\boldsymbol{\Pi}(\boldsymbol{x})+\varepsilon \boldsymbol{x}^{2 n+p+1}+\varepsilon^{\prime} \boldsymbol{x}^{2 n+p+2}+\cdots
$$

Il en résulte qu'en faisant: $\Pi_{1}(x)=\Pi(-x)$, nous aurons aux termes près de l'ordre $2 n+p+1$ :

$$
e^{x}=\frac{\Pi(x)}{\Phi(x)}, \quad e^{-x}=\frac{\Pi_{1}(x)}{\Phi(x)}
$$

et il suffira de changer $x$ en $i x$ pour retrouver sous forme réelle, les expressions que j'ai eues d'abord en vue:

$$
\cos x=\frac{S}{R}, \quad \sin x=\frac{T}{R} .
$$

Or ces polynômes $\Phi(x)$ et $\Pi(x)$ dont la considération me semble indispensable pour approfondir la question arithmétique difficile que j'ai seulement touchée, s'obtiennent comme il suit.

J'applique la formule:

$$
\int \boldsymbol{F}(t) e^{-t x} d t=-e^{-t x} \mathfrak{F}(t)
$$

où $F(t)$ est une fonction entière el $\mathfrak{F}(t)$ la quantité:

$$
\mathfrak{F}(t)=\frac{F(t)}{x}+\frac{F^{\prime}(t)}{x^{2}}+\frac{F^{\prime \prime}(t)}{x^{3}}+\cdots
$$

à la détermination de l'intégrale définie $\int_{0}^{1} t^{n}\left(1-t^{2}\right)^{p} e^{-t x} d t$. Pour cela je remarque que la relation:

$$
\int_{0}^{1} F(t) e^{-t x} d t=\mathfrak{F}(0)-e^{-x} \mathfrak{F}(1)
$$

met en évidence deux termes, dont le premier se calcule au moyen du développement:

$$
F(t)=t^{n}\left(1-t^{2}\right)^{p}=t^{n}-\frac{p}{1} l^{n+2}+\frac{p(p-1)}{1.2} t^{n+4}-\cdots+(-1)^{p} t^{n+2 p}
$$

qui donne les valeurs des dérivées de $F(t)$ pour $t=0$, on a donc immédiatement:

$$
\begin{gathered}
\mathfrak{F}(0)=\frac{1.2 .3 \ldots n}{x^{n+1}}-\frac{p}{1} \\
=\frac{1.2 .3 \ldots n+2}{x^{n+3}}+\cdots+(-1)^{p} \frac{1.2 .3 \ldots n+2 p}{x^{n+2 p+1}} \\
=\frac{1.2 .3 \ldots n}{x^{n+2} p+1} \Phi(x)
\end{gathered}
$$


en posant:

$\Phi(x)=x^{2 n}-\frac{p}{1}(n+1)(n+2) x^{2 n-2}+\frac{p(p-1)}{1.2}(n+1)(n+2)(n+3)(n+4) x^{2 n-4}-\cdots$

Soit en second lieu $t=1+h$, les dérivées de $F(t)$ pour $t=1$ s'obliendront en développant suivant les puissances de $h$ la quantité:

Faisons:

$$
F(1+h)=(-1)^{p} h^{p}(1+h)^{n}(2+h)^{p} .
$$

$$
(1+h)^{n}(2+h)^{p}=A+B h+C h^{2}+\cdots+h^{n+p},
$$

et l'on en conclura semblablement

$$
\mathfrak{F}(1)=\frac{(-1)^{p} \cdot 1 \cdot 2 \cdot 3 \ldots p}{x^{n+2 p+1}} \Pi(x)
$$

en écrivant pour abréger:

$$
\Pi(x)=A x^{n+p}+p B x^{n+p-1}+p(p+1) C x^{n+p-2}+\cdots .
$$

Ceci posé, et en observant que l'intégrale $\int_{0}^{1} t^{n}\left(1-t^{2}\right)^{p} e^{-t x} d t$ peut ètre évidemment développée sous la forme: $\varepsilon+\varepsilon_{1} x+\varepsilon_{2} x^{2}+\cdots$ la relation à laquelle nous sommes amenés à savoir:

$$
\frac{1.2 .3 \ldots n}{x^{n+2 p+1}} \Phi(x)-e^{-x} \frac{(-1)^{p 1} \cdot 2.3 \ldots p}{x^{n+2 p+1}} \Pi(x)=\varepsilon+\varepsilon_{1} x+\varepsilon_{2} x^{2}+\cdots
$$

donne facilement:

$$
e^{x} \Phi(x)-(-1)^{p} \frac{1.2 .3 \ldots p}{1.2 .3 \ldots n} \Pi(x)=\varepsilon^{\prime} x^{n+2 p+1}+\varepsilon^{\prime \prime} x^{n+2 p+2}+\cdots .
$$

Les polynômes cherchés sont donc ainsi obtenus d'une manière générale, mais . je n'en ai pas jusqu'ici fait l'étude approfondie. J'ai seulement remarqué que l'intégrale définie $\int_{0}^{1} t^{n}\left(1-t^{2}\right)^{p} e^{-t x} d t$, et ces deux autres:

$$
\int_{0}^{-1} t^{n}\left(1-t^{2}\right)^{p} e^{-t x} d t, \int_{0}^{\infty} t^{n}\left(1-t^{2}\right)^{p} e^{-t x} d t
$$

satisfont à l'équation linéaire du troisième ordre

$$
\frac{d^{3} y}{d x^{3}}-(n+2 p+3) x \frac{d^{2} y}{d x^{2}}+\frac{d y}{d x}+(n+1) x y=0 .
$$

Vannes (Morbihan) 9 Juin 1873. 